\title{
Electron-Beam Irradiation Induced Regulation of Surface Defects in Lead Halide Perovskite Thin Films
}

\author{
Binbin Jin, ${ }^{1,2}$ Ding Zhao $\mathbb{D}^{1,2}$ Fei Liang, ${ }^{3}$ Lufang Liu, ${ }^{4}$ Dongli Liu $\mathbb{D}^{1,2}$ Pan Wang, \\ and Min Qiu (iD) \\ ${ }^{1}$ Key Laboratory of 3D Micro/Nano Fabrication and Characterization of Zhejiang Province, School of Engineering, \\ Westlake University, 18 Shilongshan Road, Hangzhou, 310024 Zhejiang Province, China \\ ${ }^{2}$ Institute of Advanced Technology, Westlake Institute for Advanced Study, 18 Shilongshan Road, Hangzhou, \\ 310024 Zhejiang Province, China \\ ${ }^{3}$ State Key Laboratory of Crystal Materials and Institute of Crystal Materials, Shandong University, Jinan 250100, China \\ ${ }^{4}$ State Key Laboratory of Modern Optical Instrumentation, College of Optical Science and Engineering, Zhejiang University, \\ Hangzhou 310027, China
}

Correspondence should be addressed to Ding Zhao; zhaoding@westlake.edu.cn and Min Qiu; qiu_lab@westlake.edu.cn

Received 15 November 2020; Accepted 13 May 2021; Published 4 June 2021

Copyright (c) 2021 Binbin Jin et al. Exclusive Licensee Science and Technology Review Publishing House. Distributed under a Creative Commons Attribution License (CC BY 4.0).

\begin{abstract}
Organic-inorganic hybrid perovskites (OIHPs) have been intensively studied due to their fascinating optoelectronic performance. Electron microscopy and related characterization techniques are powerful to figure out their structure-property relationships at the nanoscale. However, electron beam irradiation usually causes damage to these beam-sensitive materials and thus deteriorates the associated devices. Taking a widely used $\mathrm{CH}_{3} \mathrm{NH}_{3} \mathrm{PbI}_{3}$ film as an example, here, we carry out a comprehensive study on how electron beam irradiation affects its properties. Interestingly, our results reveal that photoluminescence (PL) intensity of the film can be significantly improved along with blue-shift of emission peak at a specific electron beam dose interval. This improvement stems from the reduction of trap density at the $\mathrm{CH}_{3} \mathrm{NH}_{3} \mathrm{PbI}_{3}$ surface. The knock-on effect helps expose a fresh surface assisted by the surface defect-induced lowering of displacement threshold energy. Meanwhile, the radiolysis process consistently degrades the crystal structure and weaken the PL emission with the increase of electron beam dose. Consequently, the final PL emission comes from a balance between knock-on and radiolysis effects. Taking advantage of the defect regulation, we successfully demonstrate a patterned $\mathrm{CH}_{3} \mathrm{NH}_{3} \mathrm{PbI}_{3}$ film with controllable $\mathrm{PL}$ emission and a photodetector with enhanced photocurrent. This work will trigger the application of electron beam irradiation as a powerful tool for perovskite materials processing in micro-LEDs and other optoelectronic applications.
\end{abstract}

\section{Introduction}

Organic-inorganic hybrid perovskites (OIHPs) have emerged as a group of promising optoelectronic materials due to their large light absorption coefficient [1-3], high carrier mobility, and long carrier diffusion length [4-8]. The power conversion efficiency (PCE) of OIHP solar cells has been improved from $3.8 \%$ to $25.5 \%$, achieving comparable efficiency to the champion PCE of crystalline silicon-based counterparts $[9,10]$. However, OIHP such as $\mathrm{MAPbI}_{3}\left(\mathrm{MA}=\mathrm{CH}_{3} \mathrm{NH}_{3}^{+}\right)$are vulnerable to a variety of environmental factors including moisture [11-13], oxygen [14-16], heat [17, 18], and light irradiation $[19,20]$, which usually deform the crystal lattice or result in chemical decomposition. Defect passivation at the surfaces and grain boundaries (GBs) plays an important role in minimizing nonradiative recombination and maintaining stability of solution-processed polycrystalline OIHP films [21-24].

Diverse methods have been proposed to improve optoelectronic properties of OIHPs [25-31]. For instance, posttreatment in a certain humidity could enhance the PL of OIHP films, which is attributed to the partial solvation of methylammonium component and the formation of hydrogen bonding between the hydroxyl in water and uncoordinated halide ions [32]. More recently, light soaking using a standard 1-sun $\left(100 \mathrm{~mW} / \mathrm{cm}^{2}\right)$ source and continuous laser 
even ultrafast pulsed laser (nanosecond and femtosecond laser) was reported to ameliorate perovskite film quality through the uniform lattice expansion [33], the release of residual stress [34], and surface polishing [35], respectively. The coexistence of light socking and oxygen can create a favorable environment to improve optical performance (enhancement in PL intensity) of perovskites through suppression of deep trap states by transferring photo-induced electrons from perovskites to the adsorbed oxygen [36-38]. A focused electron beam (e-beam) provided in a scanning electron microscope (SEM) or transmission electron microscope (TEM) can also be used to regulate crystal structures, as well as optoelectronic properties of materials [39-41]. Although a single-crystal $\mathrm{MAPbBr}_{3}$ microplate photodetector with increased photocurrent has been fabricated through direct e-beam exposure, it is widely accepted that e-beam irradiation can significantly degrade perovskite structures as well as the PL emission [42, 43]. Therefore, how electrons affect or interact with OIHPs is still an open question.

In the present work, we comprehensively study the effect of e-beam irradiation on $\mathrm{MAPbI}_{3}$ film properties. We find that the PL intensity firstly increases and then decreases with e-beam dose. Its maximum is around sixfold larger than that of unexposed region. We determine that improved PL is due to the knock-on effect of bombarding electrons at the $\mathrm{MAPbI}_{3}$ surface, which causes exposure of a fresh surface with less trap density, while the radiolysis plays a major role in deteriorating crystal structures and thus weakens $\mathrm{PL}$ emission. It is believed that e-beam exposure at some point is helpful to attain perovskite films with higher quality, and that device performance improves accordingly.

\section{Results and Discussion}

We choose $\mathrm{MAPbI}_{3}$ as a suitable candidate to study the e-beam irradiation induced structural and compositional changes since it is a promising photovoltaic material and more sensitive to e-beam than all inorganic perovskites [43]. $\mathrm{MAPbI}_{3}$ films were prepared on ITO glass using a one-step spin-coating process (see Method). X-ray diffraction (XRD) spectrum (Figure S1a) shows dominant diffraction peaks at $14.1^{\circ}$ and $28.5^{\circ}$, which can be well indexed to (110) and (220) planes of tetragonal crystal structures. The absorption and PL spectra are shown in Figure S1b and S1c. The band edge and PL emission peak at around $762 \mathrm{~nm}$ were consistent with the previous research. Time-resolved PL (TRPL) decay profile of $\mathrm{MAPbI}_{3}$ was monitored to gain insight into charge carrier dynamic (Figure S1d), which displayed two delay lifetime of $\tau_{1}=14.7 \mathrm{~ns}$ and $\tau_{2}=134.1 \mathrm{~ns}$. All of these characterizations demonstrate the high quality of the synthesized $\mathrm{MAPbI}_{3}$ films.

A 10 by 10 square array with a side length of $4 \mu \mathrm{m}$ and a period of $10 \mu \mathrm{m}$ was exposed by a focused e-beam on the $\mathrm{MAPbI}_{3}$ film. Each square was named as MAPI $_{m-n}$, where $m$ defined the number of columns and $n$ defined the number of rows. The acceleration voltage and basic area dose were $10 \mathrm{kV}$ and $100 \mu \mathrm{C} / \mathrm{cm}^{2}$. The dose factor for the first square (named as $\mathrm{MAPI}_{1-1}$ ) was 0.5 and increased by 2.5 for the next square $\left(\right.$ dose $=$ area dose $\times$ dose factor $\left.=100 \mu \mathrm{C} / \mathrm{cm}^{2} \times[(m-1) \times 25+(n-1) \times 2.5+0.5]\right)$.

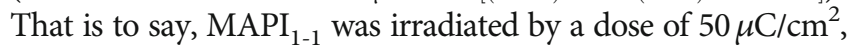
and the dose for MAPI M-10 $_{10-1}$ was increased to $24800 \mu \mathrm{C} / \mathrm{cm}^{2}$ (see details of dose distribution in Figure S2). We first examined the PL intensity of e-beam-irradiated MAPI samples. $532 \mathrm{~nm}$ laser with a low power density of $0.2 \mathrm{~mW} / \mathrm{cm}^{2}$ was employed to reduce photodamage. To our surprise, the PL intensity increases rapidly with the increase of irradiation dose (Figure 1(a)). $\mathrm{MAPI}_{6-4}$ displayed the largest PL emission (Figure 1(e)), which was around sixfold increase relative to that of unexposed sample $\left(\mathrm{MAPI}_{0}\right)$. However, as the dose continues increasing, the PL intensity begins to attenuate and it is finally lower than that of $\mathrm{MAPI}_{0}$. The detailed PL emission spectra of $\mathrm{MAPI}_{1-1}$, $\mathrm{MAPI}_{2-2}$ to $\mathrm{MAPI}_{10-10}$ (along the diagonal of array) are shown in Figure S3.

Interestingly, $\mathrm{PL}$ intensity at the edge of the square before $\mathrm{MAPI}_{6-4}$ is weaker than that at the center, while after MAPI 6 -4, the edge is higher than the center (Figure 1(a), also see Figure S4). This is due to the proximity effect in electronbeam lithography (EBL), where the actual exposure dose at the center of the pattern is higher [44, 45]. This phenomenon is consistent with the PL intensity of various squares changing with the e-beam dose. Meanwhile, the PL emission peak demonstrates a successive blue shift from $762 \mathrm{~nm}$ to $752 \mathrm{~nm}$ and gradually stabilized at $750 \mathrm{~nm}$ (Figure 1(b)). Specifically, as shown in Figures 1(c) and 1(d), the PL emission peak blue-shifts sharply from $762 \mathrm{~nm}$ $\left(\mathrm{MAPI}_{0}\right)$ to $753.7 \mathrm{~nm}\left(\mathrm{MAPI}_{4-1}\right)$, then moves slowly to $752 \mathrm{~nm}\left(\mathrm{MAPI}_{9-1}\right)$, and eventually stabilizes at $750 \mathrm{~nm}$. The enhanced PL intensity together with the blue-shift of PL emission peak implies a suppressed nonradiative recombination due to the reduced surface defects of the MAPI films [46-50].

To fully explore the interaction between e-beam and MAPI films, we carefully analyzed the structural and compositional changes of the MAPI films after e-beam exposure. Figure 2(a) shows the optical micrography of MAPI films irradiated by varying e-beam dose. As the optical reflection is heavily dependent on the film thickness, various colors will be controllably produced depending on the exposure dose. The relationship between the thinning of the MAPI film thickness and the e-beam dose will be explained in detail later. Direct writing of structural colors together with the high spatial resolution afforded by EBL has many potential applications in optical anticounterfeiting, fade-resistant color printing, and colorimetric sensing [51-54]. To investigate the dependence of surface morphology of MAPI films on the dose, top-view and cross-sectional SEM images were conducted. As illustrated in Figure 2(b), the drawn patterns of the 10 by 10 array can be clearly seen by the contrast of the image, which display brighter colors. $\mathrm{MAPI}_{0}$ without ebeam treatment is smooth with crystal grains densely packed (Figure 2(c)). We have selected three representative regions, $3 \times 3$ arrays centered at $\mathrm{MAPI}_{2-2}$ (initial stage, Figure $2(\mathrm{~d})$ ), $\mathrm{MAPI}_{6-4}\left(\mathrm{PL}_{\text {max }}\right.$, Figure 2(e)), and $\mathrm{MAPI}_{9-9}$ (final stage, Figure 2(f)), and marked with red, green, and blue box in Figure 2(b) for detail analysis. As shown in Figure 2(g), 


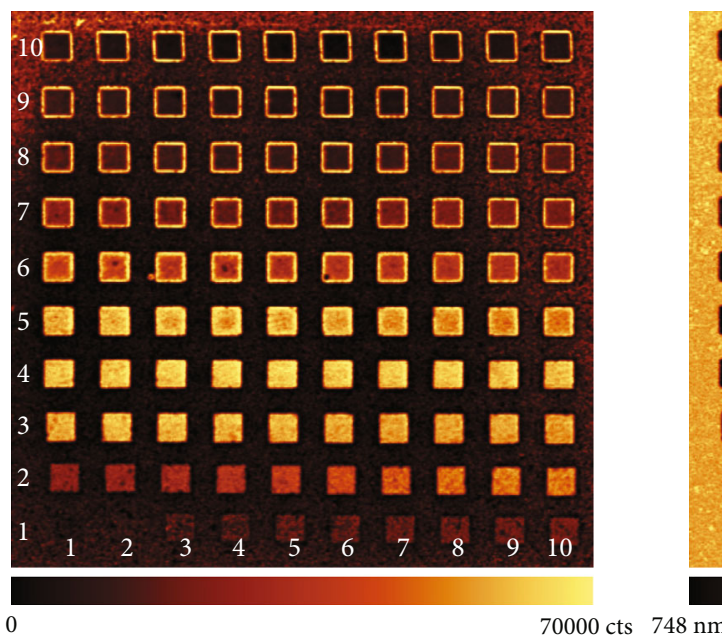

(a)

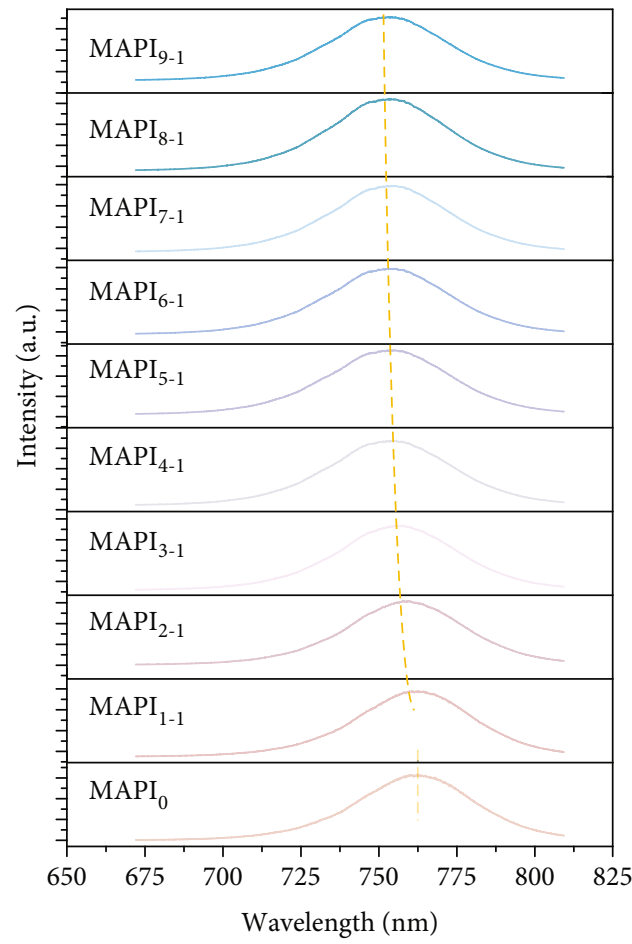

(c)

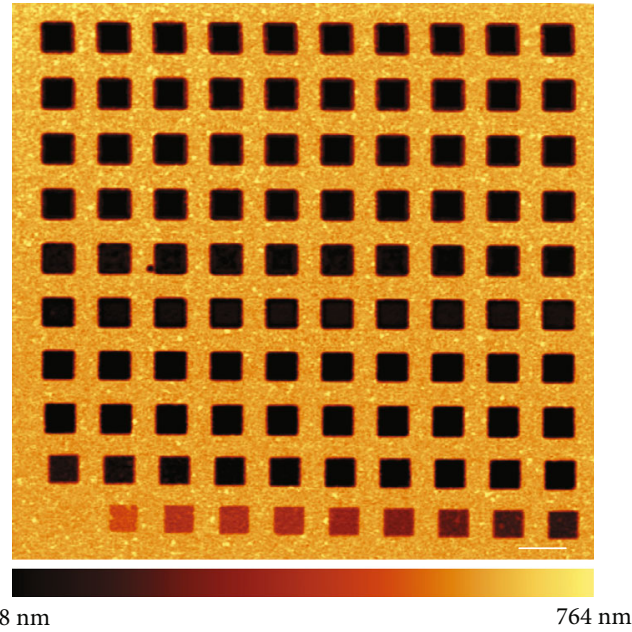

(b)

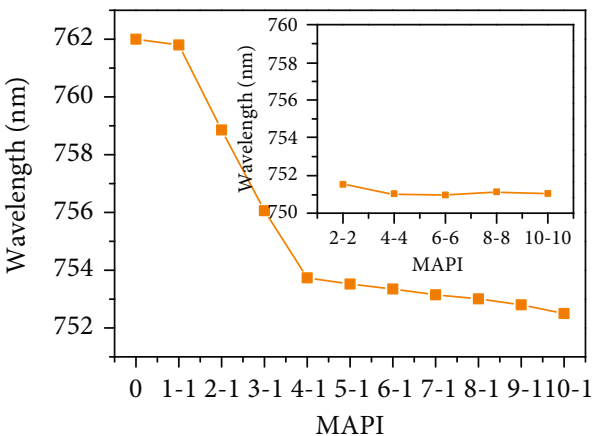

(d)

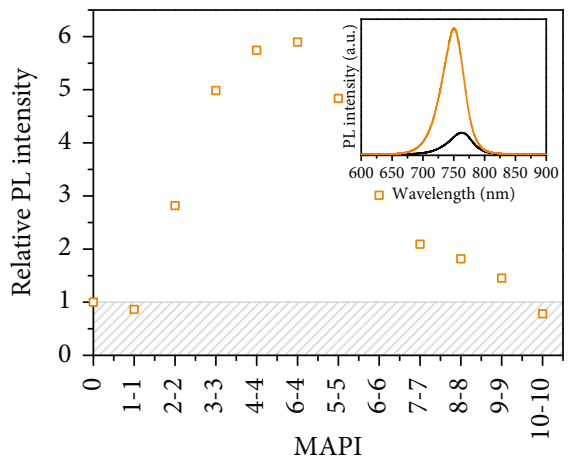

(e)

FIgURE 1: PL intensity of MAPI films irradiated by e-beam with various dose. (a) PL intensity mapping of MAPI arrays irradiated by e-beam,

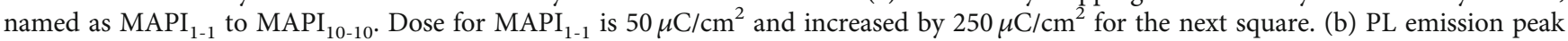
mapping of MAPI $_{1-1}$ to MAPI ${ }_{10-10}$. Scale bar: $5 \mu \mathrm{m}$. (c) PL emission spectra of MAPI M $_{0}$ to MAPI ${ }_{9-1}$. (d) Relationship between PL emission

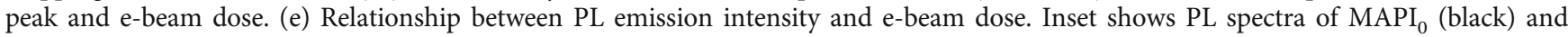
MAPI $_{6-4}$ (orange).

cracks begin to appear along the grain boundaries and then extended across the full exposure area (Figures $2(\mathrm{~h})$ and 2(i)), leaving numerous smaller grains. The width of the cracks can be extended to $c a$. $20 \mathrm{~nm}$, which will impede charge carrier transport and thus deteriorate device performance. Furthermore, atomic force microscope (AFM) was conducted to measure the height profile and roughness of the e-beam treated films. As shown in Figure S4, AFM images reveal the thinning of the MAPI films upon e-beam exposure. The thickness of the as-prepared MAPI films is about $330 \mathrm{~nm}$ (Figure S5). The thickness thinning of MAPI $_{2-2}, \mathrm{MAPI}_{6-4}$, and $\mathrm{MAPI}_{9-9}$ is about $25 \mathrm{~nm}, 30 \mathrm{~nm}$, and $45 \mathrm{~nm}$ (Figure S4b-g), respectively. The gradual thinning of MAPI films leads to the blue shift of the color displayed in optical microscope as mentioned above. Moreover, the roughness of these three regions is $8.3 \mathrm{~nm}$, 


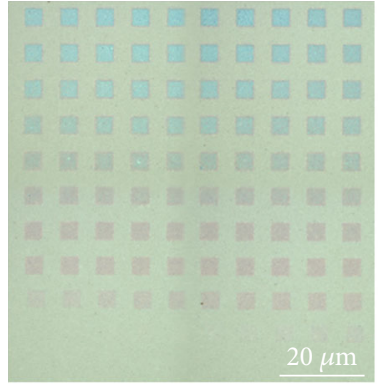

(a)

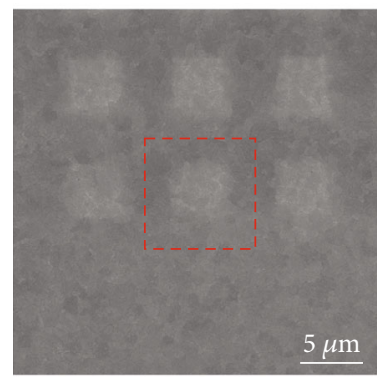

(d)

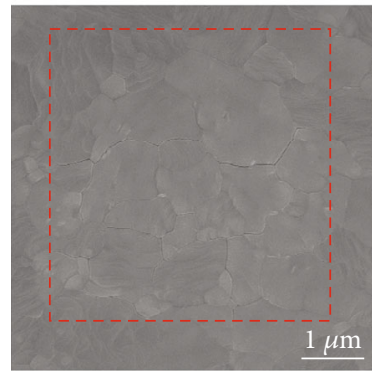

(g)

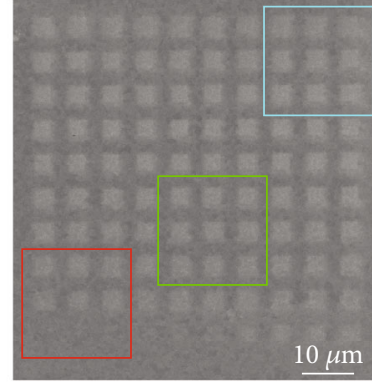

(b)

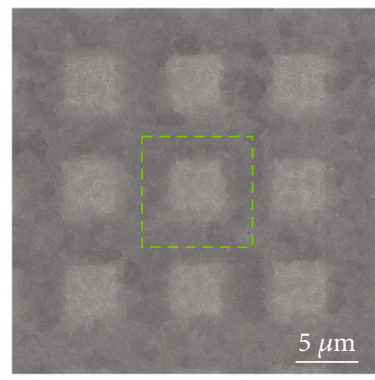

(e)

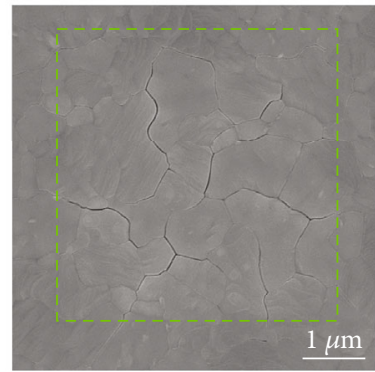

(h)

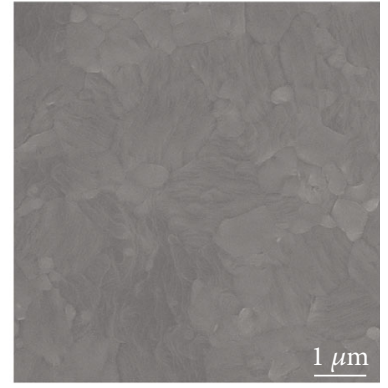

(c)

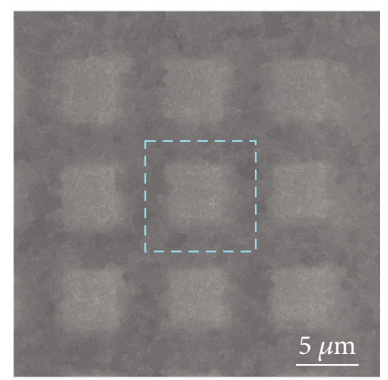

(f)

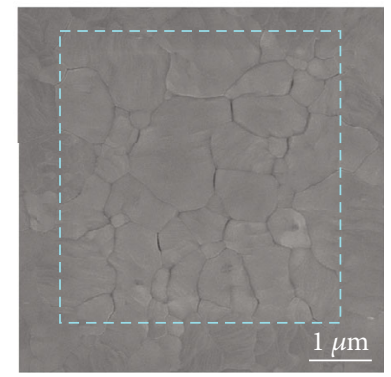

(i)

FIGURE 2: Morphological changes of MAPI films upon e-beam irradiation. (a) Optical image of MAPI 1-1 $_{\text {to MAPI }}$ 10-10. MAPI shows different colors with various e-beam dose treatment. (b) SEM image of $\mathrm{MAPI}_{1-1}$ to MAPI $\mathrm{I}_{10-10} .3 \times 3$ arrays centered at MAPI $2-2, \mathrm{MAPI}_{6-4}$, and MAPI ${ }_{9-9}$ are marked with red, green, and blue box. (c) SEM image of MAPI $_{0}$ with grains densely packed. (d-f) Corresponding magnified SEM images of arrays shown in (b). (g-i) Corresponding magnified SEM images of MAPI ${ }_{2-2}$, MAPI $_{6-4}$, and MAPI $_{9-9}$.

$9.4 \mathrm{~nm}$, and $9.5 \mathrm{~nm}$, which is almost the same as that of MAPI $_{0}(8.2 \mathrm{~nm})$. These changes in morphology provide evidence for the e-beam-driven directional "etching" of the MAPI films, which may have potential applications in fabrication of micro/nanostructured perovskite films.

A thorough analysis of the compositional change of MAPI films upon e-beam exposure was carried out to further explore the underlying mechanism of the enhanced $\mathrm{PL}$ emission. The energy-dispersive X-ray spectroscopy (EDS) was firstly used to record the element content of MAPI films (Figure S6). The $\mathrm{I} / \mathrm{Pb}$ ratio of $\mathrm{MAPI}_{0}$ is $60.7 / 18.8$, which agrees well with the stoichiometry of $\mathrm{PbI}_{3}$. Upon e-beam irradiation, $\mathrm{I}$ and $\mathrm{Pb}$ intensity do not change appreciably, with $\mathrm{I} / \mathrm{Pb}$ ratio of $60.4 / 19.2,60.5 / 19.4$, 59.9/19.4, 61.4/18.2, and 60.0/19.5 for MAPI ${ }_{1-1}, \mathrm{MAPI}_{3-3}$, $\mathrm{MAPI}_{5-5}, \mathrm{MAPI}_{7-7}$, and $\mathrm{MAPI}_{9-9}$, respectively, suggesting that the $\mathrm{I} / \mathrm{Pb}$ ratio is almost independent of the e-beam dose. We then carried out high-resolution thin-film X-ray diffractometer (HRXRD) for MAPI films to study the effect of ebeam irradiation on the crystal structure. Figure 3(a) shows three prominent peaks at $14.1^{\circ}, 28.5^{\circ}$, and $31.9^{\circ}$, corresponding to the (110), (220), and (310) planes, which demonstrate robust tetragonal phase of MAPI films. Intensities of these three peaks are also well preserved as shown in Figure 3(b). It is noteworthy that $\mathrm{MAPbI}_{3}$ would suffer from thermal degradation and convert into $\mathrm{PbI}_{2}$ upon temperature increase. The absence of $\mathrm{PbI}_{2}$-related peaks in the XRD spectra (at scattering angle of $12.7^{\circ}$ ) indicates that the thermal effect is negligible in our experiments. Combining EDS and XRD measurement, we conclude that the bulk composition and crystal structure of MAPI films have not changed upon e-beam exposure in our case.

We then carried out X-ray photoelectron spectroscopy (XPS) to explore the composition on the surface of MAPI films. Figures $3(\mathrm{c})-3(\mathrm{j})$ show the evolution of XPS $\mathrm{Pb} 4 \mathrm{f}$ and $\mathrm{C} 1 \mathrm{~s}$ core-level spectra as a function of e-beam dose. Two-sharp peaks pointing to $\mathrm{Pb} 4 \mathrm{f}_{7 / 2}$ at $138.0 \mathrm{eV}$ and $\mathrm{Pb}$ $4 \mathrm{f}_{5 / 2}$ at $143.0 \mathrm{eV}$ in $\mathrm{MAPI}_{0}$ can be assigned to the $\mathrm{Pb}^{2+}$ (Figure $3(\mathrm{c})$ ). The $\mathrm{Pb} 4 \mathrm{f}$ peaks are well preserved in $\mathrm{MAPI}_{2-2}$ (Figure 3(d)). However, the continuous e-beam exposure 


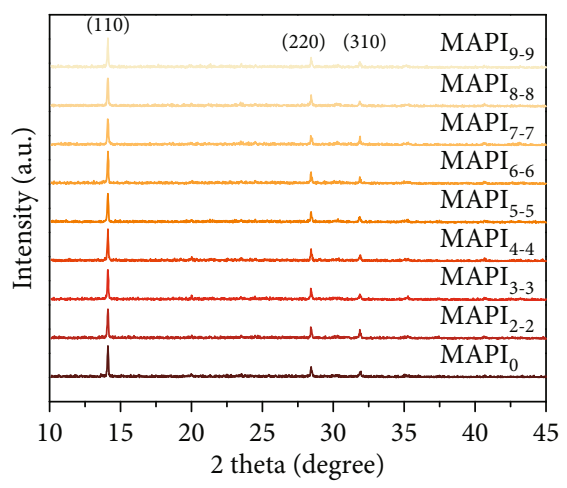

(a)

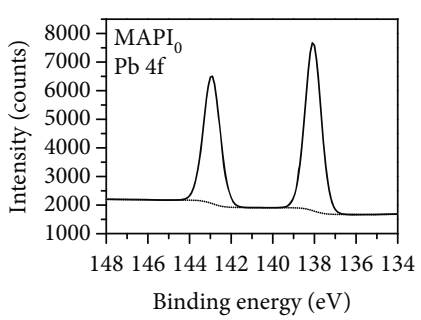

(c)

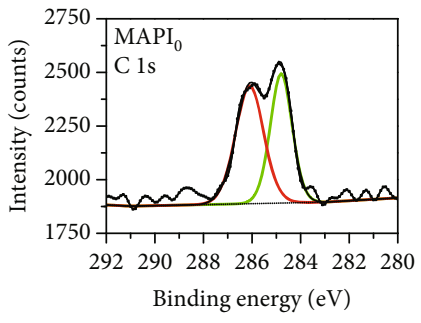

(g)

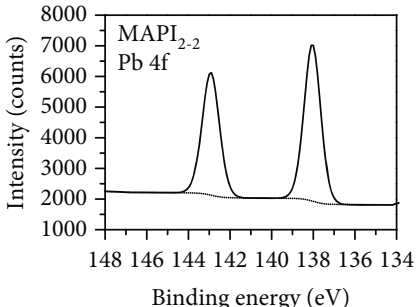

(d)

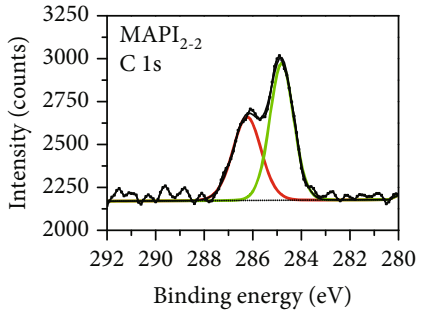

(h)

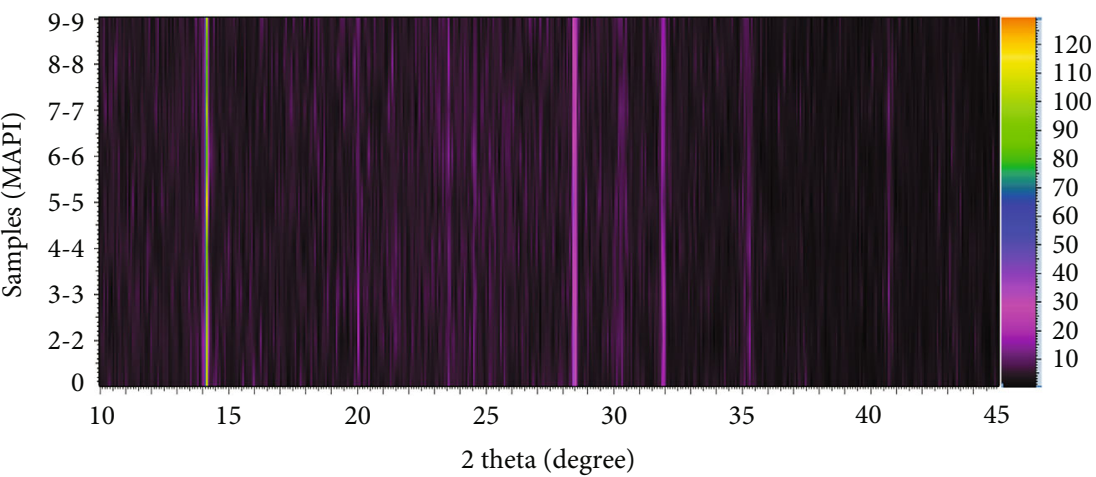

(b)

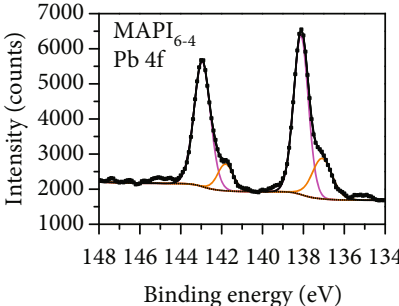

(e)

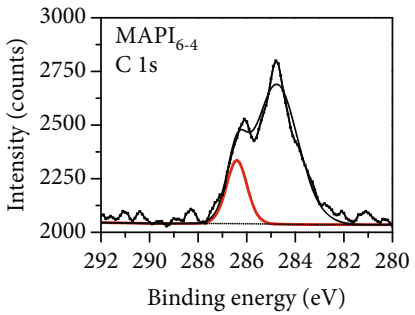

(i)

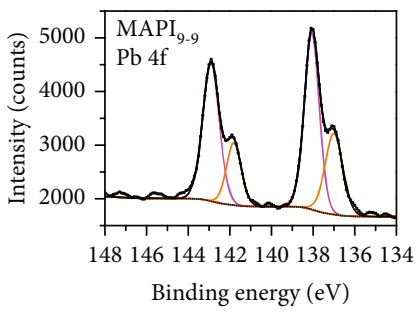

(f)

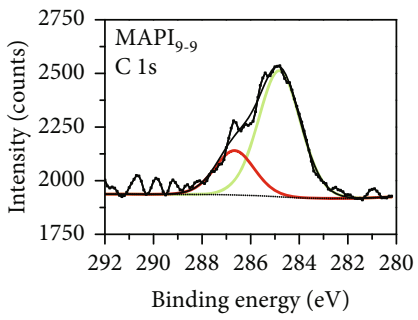

(j)

FIGURE 3: XRD and XPS Characterizations. (a) XRD patterns of MAPI $, \mathrm{MAPI}_{2-2}, \mathrm{MAPI}_{3-3}, \mathrm{MAPI}_{4-4}, \mathrm{MAPI}_{5-5}, \mathrm{MAPI}_{6-6}, \mathrm{MAPI}_{7-7}, \mathrm{MAPI}_{8-8}$, and $\mathrm{MAPI}_{9-9}$. (b) Corresponding contour map of XRD patterns. (c-f) Pb 4 f spectra of MAPI ${ }_{0}, \mathrm{MAPI}_{2-2}, \mathrm{MAPI}_{6-4}$, and MAPI ${ }_{9-9}$. (g-j) C 1s spectra of $\mathrm{MAPI}_{0}, \mathrm{MAPI}_{2-2}, \mathrm{MAPI}_{6-4}$, and $\mathrm{MAPI}_{9-9}$.

caused the reduction of $\mathrm{Pb}^{2+}$ to metallic $\mathrm{Pb}$ in $\mathrm{MAPI}_{6-4}$ with the appearance of $\mathrm{Pb}^{0}$ peaks (Figure 3(e)). The decreased and enhanced intensities of $\mathrm{Pb}^{2+}$ and $\mathrm{Pb}^{0}$ peaks as the dose increased to $\mathrm{MAPI}_{9-9}$ (Figure 3(f)) suggest the persistent reduction of $\mathrm{Pb}^{2+}$ to metallic $\mathrm{Pb}$ through radiolysis. Figures 3(g)-3(j) show the C 1 s core-level spectra of $\mathrm{MAPI}_{0}$, $\mathrm{MAPI}_{2-2}, \mathrm{MAPI}_{6-4}$, and $\mathrm{MAPI}_{9-9}$, respectively. The higher binding energy at $286.3 \mathrm{eV}$ represents the $\mathrm{C}-\mathrm{N}$ bond attributed to $\mathrm{CH}_{3} \mathrm{NH}_{3}$ cation, and the other lower binding energy at $284.8 \mathrm{eV}$ results from the absorbed ex situ hydrocarbons (e.g., C-C, C-H) [55]. It is obvious that the peak intensity of $\mathrm{C}-\mathrm{N}$ bond gradually diminishes with increasing e-beam dose, suggesting the persistent decomposition of $\mathrm{CH}_{3} \mathrm{NH}_{3}$ cation.

Till now, the experimental results gradually reveal the interaction between the e-beam and MAPI films. It is known that knock-on damage and radiolysis usually occur during electron-matter interaction [42, 43]. When the electron energy is beyond a certain threshold energy for the displacement $\left(E_{\mathrm{d}}\right)$ of a particular atom, knock-on damage and surface sputtering will happen. Radiolysis (or ionization damage) involves inelastic electron-electron or electron-phonon scat- tering that drives atomic displacement through energymomentum transfer assisted by either thermal vibrations or Coulomb interactions. In previous studies, e-beam induced radiolysis has been considered as the major mechanism in perovskite materials since the incident electron energy is usually below the $E_{\mathrm{d}}$ [41]. In reality, such a threshold can be significantly lowered because of the finite sample size (weak bonds of surface and edge atoms) and the existence of defects [56]. Therefore, the knock-on effect cannot be neglected. In this study, considering the soft ionic nature of MAPI films, various defects such as point defects (i.e., vacancies, interstitials, and antisites) and two-dimensional defects (grain boundaries) are generated unavoidably due to the fast annealing crystallization process and solution-processed stoichiometric issues [57]. Moreover, the trap states are 1-2 orders of magnitude greater than that of the film interior [58]. We calculated the activation energies for the displacement of halide ions on MAPI surfaces with and without halide vacancies $\left(V_{\mathrm{I}}\right)$. The activation energy for I ion displacement in defect-free MAPI surface was $4.05 \mathrm{eV}$; whereas a lower activation energy of $3.49 \mathrm{eV}$ was found on $V_{\mathrm{I}}$ surface 


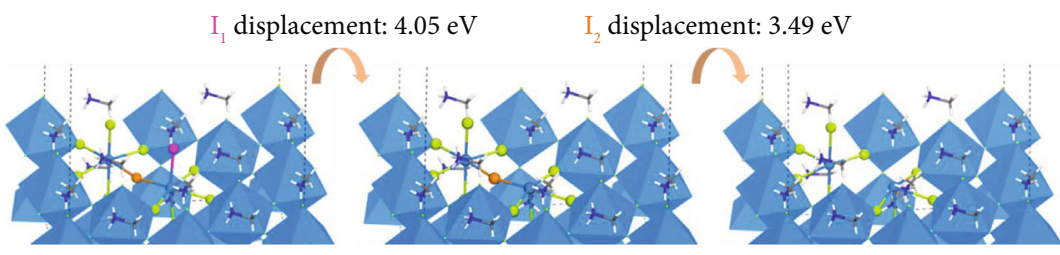

(a)
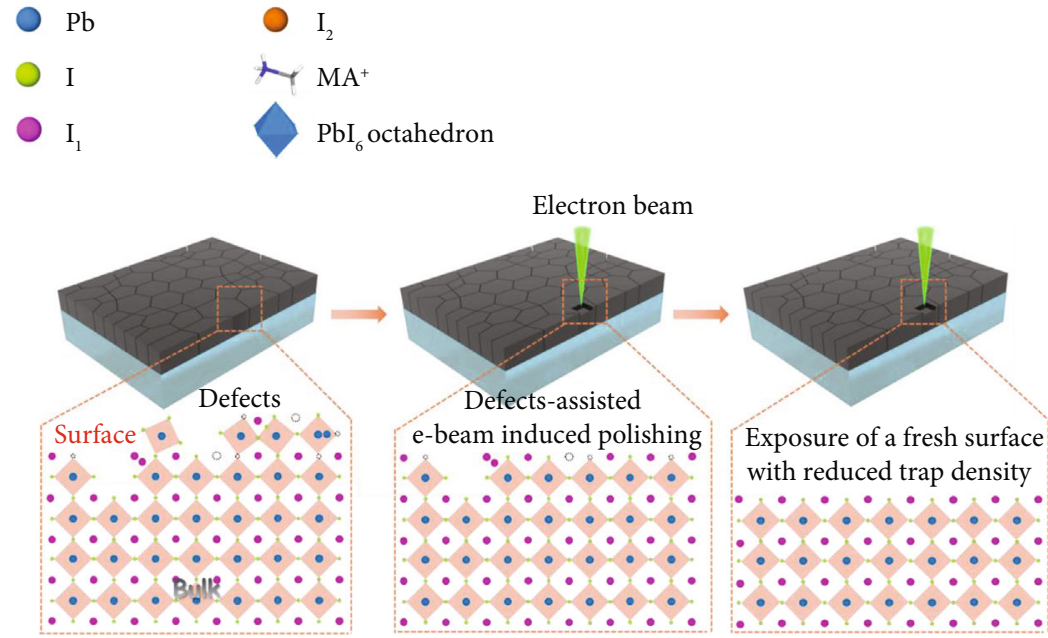

(b)

FIgURE 4: The mechanism for the regulation of surface defects of MAPI films via e-beam irradiation. (a) Calculated activation energies for I ion displacement $\left(E_{\mathrm{a}}\right)$ on perfect slab and $V_{\mathrm{I}}$ slab, $E_{\mathrm{a}}$ for $\mathrm{I}_{1}$ and $\mathrm{I}_{2}$ are $4.05 \mathrm{eV}$ and $3.49 \mathrm{eV}$. (b) A schematic illustration for the regulation of surface defects, e-beam induced thinning of MAPI films through knock-on assisted by surface defects.

(Figure 4(a)). This indicates that $V_{\mathrm{I}}$ could reduce the threshold for the following atomic displacement. We speculate that defect-assisted knock-on might first take place during ebeam exposure, just like the ripple effect, leading to the thinning of MAPI film and the emergence of underlying fresh MAPI (Figure 4(b)). The fresh surface with much less defect density than initial MAPI surface contributed to the enhanced PL emission and blue-shifted emission peak. Meanwhile, the radiolysis induced decomposition of C-N bond and reduction of $\mathrm{Pb}^{2+}$ to metallic $\mathrm{Pb}$ was accompanied with knock-on, which deteriorated MAPI crystal structures and thus weakened PL emission. It is notable that both knock-on and radiolysis are thought to be dependent only on total dose received and independent on dose rate. Therefore, the final PL emission is the result of the balance of these two effects. With increasing the e-beam dose from $\mathrm{MAPI}_{0}$ to $\mathrm{MAPI}_{6-4}$, knock-on is stronger than radiolysis, leading to the persistent increasing in PL emission. However, the radiolysis plays a dominant role with continuously increasing dose; as a result, the $\mathrm{PL}$ intensity begins to decrease and finally lowers than that of $\mathrm{MAPI}_{0}$. The efficiency of radiolytic damage is strongly temperature dependent, and the damage rate can be significantly reduced when cooling the specimen. In the future, we will further explore the PL emission of MAPI films upon e-beam irradiation at low temperature.

Taking advantage of e-beam regulating surface defects, we demonstrate a high-resolution patterned MAPI film with controllable PL emission. As illustrated in Figures 5(a) and 5(b), the patterns of "WESTLAKE UNIVERSITY" in Chinese and English characters have different PL intensities and peaks. The exposure doses for sites $\mathrm{A}, \mathrm{B}$, and $\mathrm{C}$ are $8800 \mu \mathrm{C} / \mathrm{cm}^{2}$ (equivalent to $\mathrm{MAPI}_{6-4}$ ), $4050 \mu \mathrm{C} / \mathrm{cm}^{2}$ (equivalent to $\mathrm{MAPI}_{7-2}$ ), and 0 (equivalent to $\mathrm{MAPI}_{0}$ ), respectively, and their PL emission spectra are shown in Figure 5(c) with an optical image inset. This patterning method has potential applications in fabricating perovskite-based micro-LED display, which is widely believed to be incompatible with typical top-down lithography [59].

The e-beam induced defect regulation in MAPI film is also applied to improve the performance of optoelectronic devices. Here, we fabricated a metal-semiconductor-metal (MSM) MAPI photodetector (PD) as an example. $60 \mathrm{~nm}$ thick gold electrodes with a $40 \mu \mathrm{m}$ electrode gap were deposited on MAPI films by shadow mask-assisted e-beam evaporation. The schematic illustration of $\mathrm{PD}$ is shown in Figure 6(a). Then, the MAPI film was partially treated through e-beam irradiation with various doses. An optical image in Figure 6(b) clearly shows exposed areas between electrode gaps. Figure 6(c) shows I-V curves of our fabricated PD under halogen light illumination with irradiance of $0.5 \mathrm{~mW} / \mathrm{cm}^{2}$. The spot radius is $c a .10 \mu \mathrm{m}$ and it is confined within the e-beam exposure region. For the photodetector without e-beam irradiation, we obtain a photocurrent of $1.0 \mathrm{nA}$ when applying a voltage of $4 \mathrm{~V}$. As expected, the photocurrent can be raised after e-beam exposure, and the maximum is about $2.0 \mathrm{nA}$ using the dose as $\mathrm{MAPI}_{5-1}$. From the SEM image in Figures 6(d) and 6(e), we see that grains 


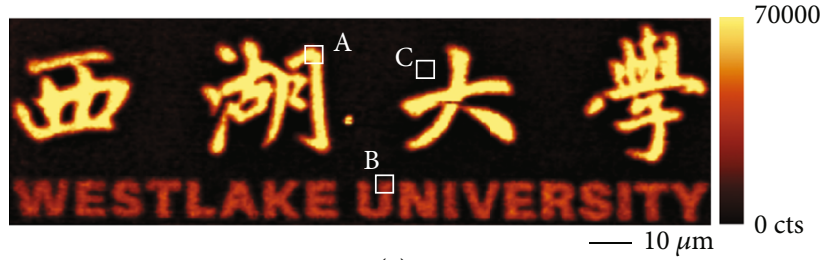

(a)

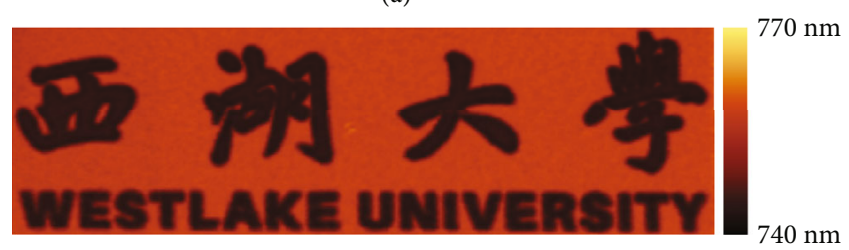

(b)

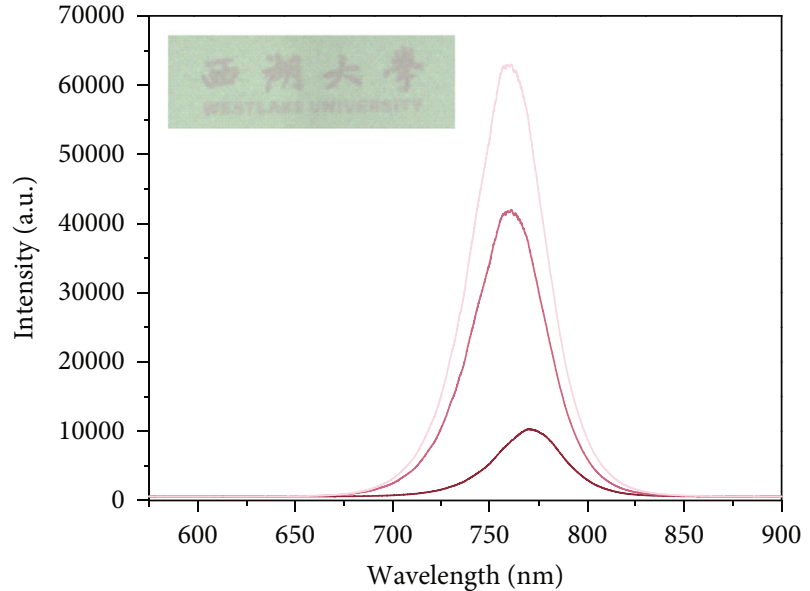

(c)

Site A
- Site B
- Site C

FIGURE 5: E-beam writing micropatterns on MAPI films. (a) PL intensity mapping of "Westlake University". The dose for sites A, B, and C is 8800, 4050, and $0 \mu \mathrm{C} / \mathrm{cm}^{2}$. (b) PL emission peak mapping of "Westlake University." (c) Corresponding PL spectra of sites A, B, and C, respectively. Inset is an optical image of "Westlake University".

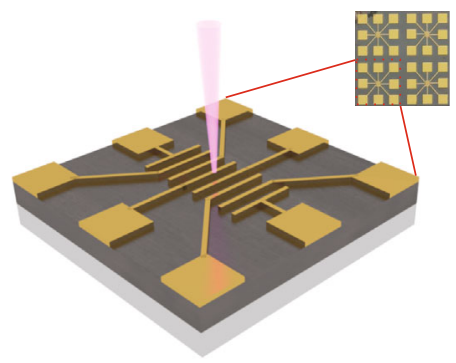

(a)

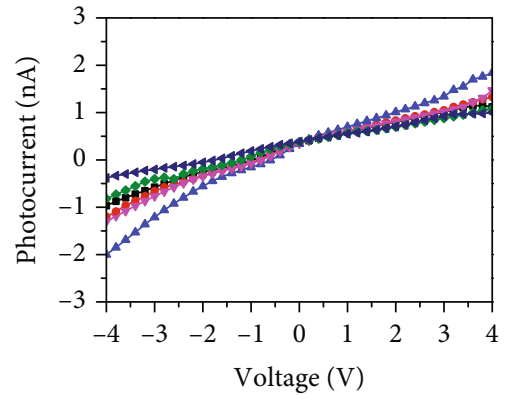

(c)

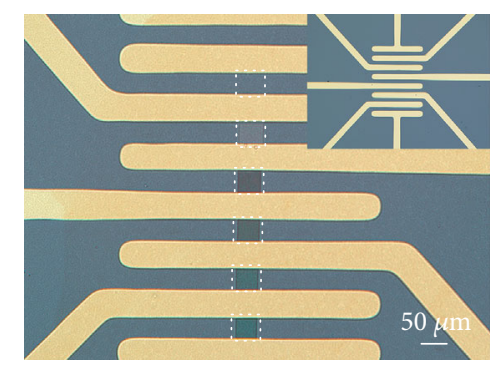

(b)

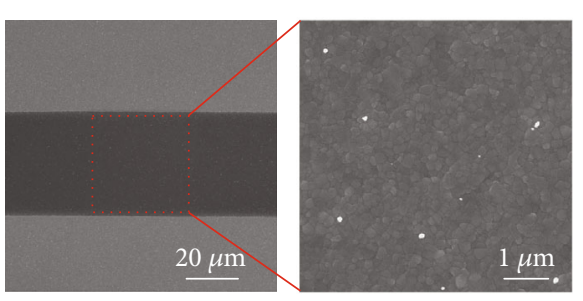

(d)

(e)

$$
\begin{array}{ll}
\rightarrow \text { MAPI }_{0} & \rightarrow \text { MAPI }_{2-2} \\
\rightarrow \text { MAPI }_{1-1} & \rightarrow \text { MAPI }_{6-4} \\
\simeq \text { MAPI }_{5-1} & \leftarrow \text { MAPI }_{9-9}
\end{array}
$$

FIGURE 6: Structure and characteristics of fabricated MAPI photodetectors. (a) Schematic illustration of the photodetector. (b) Optical image of the MAPI photodetector upon e-beam irradiation (marked with dashed line box). Inset is the original photodetector. (c) I-V characteristics of $\mathrm{MAPI}_{0}, \mathrm{MAPI}_{1-1}, \mathrm{MAPI}_{5-1}, \mathrm{MAPI}_{2-2}, \mathrm{MAPI}_{6-4}$, and $\mathrm{MAPI}_{9-9}$ with $0.5 \mathrm{~mW} / \mathrm{cm}^{2}$ halogen light illumination. (d, e) SEM image and corresponding magnified image of $\mathrm{MAPI}_{5-1}$. 
in the MAPI film are still densely packed. The improved photocurrent is attributed to the less trap states at the MAPI surface and the following decrease is due to the broadened grain boundaries which make the charge carries encounter more scattering. In terms of their being unstable in common humid air, a passivation using a hydrophobic polymer layer or thin glass encapsulation is necessary for practical applications.

\section{Conclusions}

In summary, we have experimentally investigated structural and compositional changes of MAPI films upon e-beam irradiation with various doses. Based on theories of electronmatter interaction and careful characterizations of surface morphology, crystalline, element composition, and valence, we believe that the counterintuitive PL enhancement after e-beam exposure is attributed to the emergence of a fresh surface with less defects. The e-beam regulating surface defects promotes the fabrication of a patterned MAPI film with controllable PL and a photodetector with enhanced photocurrent. This work provides an alternative way to precisely regulate and improve the performance of OIHP devices with typical electron beam lithography.

\section{Experimental Methods}

4.1. Materials. Methylammonium iodide (MAI, 99.5\%) and lead iodide $\left(\mathrm{PbI}_{2}, 99.999 \%\right)$ were purchased from Shanghai MaterWin New Materials Co., Ltd. N, N-dimethylformamide (DMF, 99.7+\%), dimethyl sulfoxide (DMSO), and Chlorobenzene were purchased from Alfa Aesar. All materials and reagents were used as received without further purification.

4.2. Synthesis MAPI Film. The MAPI film was prepared by a typical antisolvent spin-coating method. Specifically, the MAPI perovskite ink $(1.25 \mathrm{M})$ was prepared by dissolving MAI and $\mathrm{PbI}_{2}$ in a mixture solvent of DMF and DMSO $(4: 1, v / v) .40 \mu \mathrm{L}$ of MAPI precursor were dropped on the ITO glass, followed by spinning at $6000 \mathrm{rpm}$ for $30 \mathrm{~s}$. At the $6^{\text {th }}$ second of spinning, $200 \mu \mathrm{L}$ of chlorobenzene was quickly dropped to assist in forming a dense perovskite film and thermal annealing at a temperature of $100^{\circ} \mathrm{C}$ on the hotplate was to crystallize the MAPI film.

4.3. Photodetector Fabrication. MAPI films were prepared on a $100 \mathrm{~nm} \mathrm{SiO}_{2}$-covered $\mathrm{Si}$ wafer as described previously. A $60 \mathrm{~nm}$ thick gold film was deposited on perovskite surface by e-beam evaporator using a shadow mask. The gap between the electrode pairs is $40 \mu \mathrm{m}$. Then, the gap was performed with e-beam irradiation (acceleration voltage: $10 \mathrm{kV}$; current beam: $200 \mathrm{pA}$ ) with various doses.

4.4. DFT Calculations. The DFT calculations were performed by the CASTEP package [60] with the ultrasoft pseudopotentials [61] and GGA-PW91 functional [62, 63]. A $600 \mathrm{eV}$ plane wave basis sets the cutoff, and the cutoff energy was chosen in our calculations. The $3 \times 3 \times 1$ Monkhorst-Pack grids [64] of $k$-points were used for the (001) surface of tetragonal $\mathrm{MAPbI}_{3}$ phase. The convergence thresholds between optimi- zation cycles for energy change and maximum force were set as $5.0 \times 10^{-6} \mathrm{eV} /$ atom and $0.03 \mathrm{eV} / \AA$, respectively.

A stoichiometric slab of $17.68 \AA \times 17.68 \AA \times 24.84 \AA$ with a vacuum thickness of $12 \AA$ was constructed to model (001) surface of tetragonal $\mathrm{MAPbI}_{3}$ phase. The MAI-terminal was selected. In all calculations, the atoms in the bottom layers were fixed, but the atoms in the two topmost layers were allowed to relax. The formation energy of iodine defects was defined as

$$
E_{\mathrm{f}}=\left[E_{(\text {iodine })}+E_{(\text {defect slab })}\right]-E_{(\text {perfect slab })},
$$

where the first term is the total energy of isolated iodine atom, molecule, the second term is the total energy of defect slab containing iodine defect, and the third term is the total energy of the perfect slab without defect. According to above definitions, a larger $E_{\mathrm{f}}$ value represents that the iodine defect is more difficult to emerge.

4.5. Characterization. UV-vis-NIR absorption of MAPI films was recorded on the UV3600Plus spectrophotometer (Shimadzu, Japan). Steady-state photoluminescence (PL) spectra and images were measured on alpha 300R (WITec $\mathrm{GMBH}$, Germany) confocal Raman system. A diodepumped solid-state laser (532 nm, cobalt Laser) was focused on samples with a diffraction-limited beam size of $350 \mathrm{~nm}$ by a $100 \mathrm{x}$ objective $(\mathrm{NA}=0.90)$. The collected $\mathrm{PL}$ signal was dispersed by UHTS $600 \mathrm{~mm}$ spectrometer and detected using an electron-magnified charge-coupled-device (EMCCD) thermoelectrically cooled to $-60^{\circ} \mathrm{C}$. Ultrafast PL imaging was running with $100 \mathrm{~nm}$ step size and $15 \mathrm{~ms}$ integration time. The time-resolved photoluminescence spectra were recorded using standard time-correlated single-photon counting (TCSPC) Lifetime fluorescence spectrometer (FLS1000, Edinburgh, UK). The exciting light was a picosecond pulsed laser source at $405 \mathrm{~nm}$ (EPL-405, Pulse Width $46.7 \mathrm{ps}$ ). The XRD patterns were recorded on a desktop diffractometer with a $\mathrm{Cu} \mathrm{K} \alpha$ source at the range of $10^{\circ}-60^{\circ}$ (D8 Advance; Bruker, Germany). The microdomain XRD analysis was performed on Bruker D8 Discover High-Resolution X-ray diffractometer, which is equipped with point-focused rotational $\mathrm{Cu}$ anode and DUO detectors of Scintillation counter and LynxEye detector. Each individual domain was precisely localized by laserassisted camera and well aligned by procedure of $\mathrm{z}$ scan for sample height determination and rocking curve for sample surface alignment. Then, measurements were conducted by 2Theta-Omega scan or Grazing Incident Diffraction (GID). XPS was performed on ESCLAB Xi+ (Thermo Fisher), using a monochromatized X-ray source (Al K $\alpha, 1486.6 \mathrm{eV}$ ). The diameter of the incident X-ray spot was $200 \mu \mathrm{m}$. Analysis area of $100 \mu \mathrm{m}$ was obtained by reducing apertures. Location of microdomain was confirmed by parallel imaging. Energy calibration was performed by fixing the $\mathrm{C}-\mathrm{C}$ component of $\mathrm{C}$ 1s spectrum at $284.8 \mathrm{eV}$. SEM images and EDS analysis of the samples were obtained on a field emission scanning electron 
microscope (Crossbeam550L, Zeiss) with an accelerating voltage of $10 \mathrm{kV}$.

\section{Data Availability}

All data are available in the manuscript, supplementary materials, or from the author.

\section{Conflicts of Interest}

The authors declare no competing interests.

\section{Acknowledgments}

The authors gratefully acknowledge the support from the National Natural Science Foundation of China (61927820 and 62005226) and the China Postdoctoral Science Foundation (2020M671808, 2020M671810, and 2020T130602).

\section{Supplementary Materials}

Figure S1: (a) XRD pattern, (b) UV-vis absorbance spectrum, (c) steady PL spectrum, and (d) time-resolved PL spectrum of MAPI0 sample. Figure S2: distribution of E-beam dose factor from MAPI1-1 to MAPI10-10. Figure S3: steady PL spectra of MAPI0, MAPI1-1, MAPI2-2, MAPI3-3, MAPI4-4, MAPI5-5, MAPI6-6, MAPI7-7, MAPI8-8, MAPI9-9, and MAPI10-10, respectively. Figure S4: PL intensity extract from (a) MAPI6-2, MAPI7-2, and MAPI8-2 and (b) MAPI6-8, MAPI7-8, and MAPI8-8. Figure S5: AFM image of (a) MAPI0, three representative $3 * 3$ array centered at (b) MAPI2-2, (c) MAPI6-4, and (d) MAPI9-9. Height profile of (e) MAPI2-2 marked in (b), (f) MAPI6-4 marked in (c), and (g) MAPI9-9 marked in (d). Figure S6: crosssectional SEM image of MAPI film coated on ITO glass. (Supplementary Materials)

\section{References}

[1] A. Rajagopal, K. Yao, and A. K. Y. Jen, "Toward perovskite solar cell commercialization: a perspective and research roadmap based on interfacial engineering," Advanced Materials, vol. 30, no. 32, article e1800455, 2018.

[2] J. Huang, Y. Yuan, Y. Shao, and Y. Yan, "Understanding the physical properties of hybrid perovskites for photovoltaic applications," Nature Reviews Materials, vol. 2, no. 7, p. 17042, 2017.

[3] N.-G. Park and K. Zhu, "Scalable fabrication and coating methods for perovskite solar cells and solar modules," Nature Reviews Materials, vol. 5, no. 5, pp. 333-350, 2020.

[4] W. A. Dunlap-Shohl, Y. Zhou, N. P. Padture, and D. B. Mitzi, "Synthetic approaches for halide perovskite thin films," Chemical Reviews, vol. 119, no. 5, pp. 3193-3295, 2019.

[5] B. Yang, O. Dyck, J. Poplawsky et al., "Perovskite solar cells with near $100 \%$ internal quantum efficiency based on large single crystalline grains and vertical bulk heterojunctions," Journal of the American Chemical Society, vol. 137, no. 29, pp. 9210-9213, 2015.

[6] E. M. Tennyson, T. A. S. Doherty, and S. D. Stranks, "Heterogeneity at multiple length scales in halide perovskite semicon- ductors," Nature Reviews Materials, vol. 4, no. 9, pp. 573-587, 2019.

[7] D. Shi, V. Adinolfi, R. Comin et al., "Solar cells. Low trap-state density and long carrier diffusion in organolead trihalide perovskite single crystals," Science, vol. 347, no. 6221, pp. 519-522, 2015.

[8] S. D. Stranks, G. E. Eperon, G. Grancini et al., "Electron-hole diffusion lengths exceeding 1 micrometer in an organometal trihalide perovskite absorber," Science, vol. 342, no. 6156, pp. 341-344, 2013.

[9] A. Kojima, K. Teshima, Y. Shirai, and T. Miyasaka, "Organometal halide perovskites as visible-light sensitizers for photovoltaic cells," Journal of the American Chemical Society, vol. 131, no. 17, pp. 6050-6051, 2009.

[10] NREL National Center for Photovoltaics, "Best research-cell efficiency chart," 2020, https://www.nrel.gov/pv/cellefficiency.html.

[11] J. L. Yang, B. D. Siempelkamp, D. Liu, and T. L. Kelly, "Investigation of $\mathrm{CH}_{3} \mathrm{NH}_{3} \mathrm{PbI}_{3}$ degradation rates and mechanisms in controlled humidity environments using in situ techniques," ACS Nano, vol. 9, no. 2, pp. 1955-1963, 2015.

[12] A. M. A. Leguy, Y. Hu, M. Campoy-Quiles et al., "Reversible hydration of $\mathrm{CH} 3 \mathrm{NH} 3 \mathrm{PbI} 3$ in films, single crystals, and solar cells," Chemistry of Materials, vol. 27, no. 9, pp. 3397-3407, 2015.

[13] J. H. Noh, S. H. Im, J. H. Heo, T. N. Mandal, and S. I. Seok, "Chemical management for colorful, efficient, and stable inorganic-organic hybrid nanostructured solar cells," Nano Letters, vol. 13, no. 4, pp. 1764-1769, 2013.

[14] Q. Sun, P. Fassl, D. Becker-Koch et al., "Role of microstructure in oxygen induced photodegradation of methylammonium lead triiodide perovskite films," Advanced Energy Materials, vol. 7, no. 20, p. 1700977, 2017.

[15] N. Aristidou, I. Sanchez-Molina, T. Chotchuangchutchaval et al., "The role of oxygen in the degradation of methylammonium lead trihalide perovskite photoactive layers," Angewandte Chemie, International Edition, vol. 54, no. 28, pp. 8208-8212, 2015.

[16] Y. Ouyang, L. Shi, Q. Li, and J. Wang, "Role of water and defects in photo-oxidative degradation of methylammonium lead iodide perovskite," Small Methods, vol. 3, no. 7, p. 1900154, 2019.

[17] Y. Han, S. Meyer, Y. Dkhissi et al., "Degradation observations of encapsulated planar $\mathrm{CH}_{3} \mathrm{NH}_{3} \mathrm{PbI}_{3}$ perovskite solar cells at high temperatures and humidity," Journal of Materials Chemistry A, vol. 3, no. 15, pp. 8139-8147, 2015.

[18] S. N. Habisreutinger, T. Leijtens, G. E. Eperon, S. D. Stranks, R. J. Nicholas, and H. J. Snaith, "Carbon nanotube/polymer composites as a highly stable hole collection layer in perovskite solar cells," Nano Letters, vol. 14, no. 10, pp. 55615568, 2014.

[19] T. A. Berhe, W.-N. Su, C.-H. Chen et al., "Organometal halide perovskite solar cells: degradation and stability," Energy \& Environmental Science, vol. 9, no. 2, pp. 323-356, 2016.

[20] H. Yuan, E. Debroye, K. Janssen et al., "Degradation of methylammonium lead iodide perovskite structures through light and electron beam driven ion migration," Journal of Physical Chemistry Letters, vol. 7, no. 3, pp. 561-566, 2016.

[21] B. W. Park and S. I. Seok, "Intrinsic instability of inorganicorganic hybrid halide perovskite materials," Advanced Materials, vol. 31, no. 20, p. 1805337, 2019. 
[22] X. Zheng, B. Chen, J. Dai et al., "Defect passivation in hybrid perovskite solar cells using quaternary ammonium halide anions and cations," Nature Energy, vol. 2, no. 7, p. 17102, 2017.

[23] Q. Jiang, Y. Zhao, X. Zhang et al., "Surface passivation of perovskite film for efficient solar cells," Nature Photonics, vol. 13, no. 7, pp. 460-466, 2019.

[24] Q. Wang, Q. Dong, T. Li, A. Gruverman, and J. Huang, “Thin insulating tunneling contacts for efficient and water-resistant perovskite solar cells," Advanced Materials, vol. 28, no. 31, pp. 6734-6739, 2016.

[25] D. Bi, C. Yi, J. Luo et al., "Polymer-templated nucleation and crystal growth of perovskite films for solar cells with efficiency greater than 21\%," Nature Energy, vol. 1, no. 10, p. 16142, 2016.

[26] D. Luo, W. Yang, Z. Wang et al., "Enhanced photovoltage for inverted planar heterojunction perovskite solar cells," Science, vol. 360, no. 6396, pp. 1442-1446, 2018.

[27] W. Q. Wu, P. N. Rudd, Z. Ni et al., "Reducing surface halide deficiency for efficient and stable iodide-based perovskite solar cells," Journal of the American Chemical Society, vol. 142, no. 8, pp. 3989-3996, 2020.

[28] X. Xiao, C. Bao, Y. Fang et al., “Argon plasma treatment to tune perovskite surface composition for high efficiency solar cells and fast photodetectors," Advanced Materials, vol. 30, no. 9, p. 1705176, 2018.

[29] X. Gong, M. Li, X.-B. Shi, H. Ma, Z.-K. Wang, and L.-S. Liao, "Controllable perovskite crystallization by water additive for high-performance solar cells," Advanced Functional Materials, vol. 25, no. 42, pp. 6671-6678, 2015.

[30] T. Jeon, H. M. Jin, S. H. Lee et al., "Laser crystallization of organic-inorganic hybrid perovskite solar cells," ACS Nano, vol. 10, no. 8, pp. 7907-7914, 2016.

[31] P. You, G. Li, G. Tang, J. Cao, and F. Yan, "Ultrafast laserannealing of perovskite films for efficient perovskite solar cells," Energy \& Environmental Science, vol. 13, no. 4, pp. 1187-1196, 2020.

[32] G. E. Eperon, S. N. Habisreutinger, T. Leijtens et al., “The importance of moisture in hybrid lead halide perovskite thin film fabrication," ACS Nano, vol. 9, no. 9, pp. 9380-9393, 2015.

[33] H. Tsai, R. Asadpour, J. C. Blancon et al., "Light-induced lattice expansion leads to high-efficiency perovskite solar cells," Science, vol. 360, no. 6384, pp. 67-70, 2018.

[34] C. Song, L. Tong, F. Liu, L. Ye, and G. J. Cheng, "Addressing the reliability and electron transport kinetics in halide perovskite film via pulsed laser engineering," Advanced Functional Materials, vol. 30, no. 5, p. 1906781, 2020.

[35] W. Kong, C. Zhao, J. Xing et al., "Enhancing perovskite solar cell performance through femtosecond laser polishing," Solar $R R L$, vol. 4, no. 7, p. 2000189, 2020.

[36] Y. Tian, A. Merdasa, E. Unger et al., "Enhanced organo-metal halide perovskite photoluminescence from nanosized defectfree crystallites and emitting sites," Journal of Physical Chemistry Letters, vol. 6, no. 20, pp. 4171-4177, 2015.

[37] S. G. Motti, M. Gandini, A. J. Barker, J. M. Ball, A. R. Srimath Kandada, and A. Petrozza, "Photoinduced emissive trap states in lead halide perovskite semiconductors," ACS Energy Letters, vol. 1, no. 4, pp. 726-730, 2016.

[38] J. He, W. H. Fang, R. Long, and O. V. Prezhdo, "Why oxygen increases carrier lifetimes but accelerates degradation of $\mathrm{CH}_{3} \mathrm{NH}_{3} \mathrm{PbI}_{3}$ under light irradiation: time-domain ab initio analysis," Journal of the American Chemical Society, vol. 142, no. 34, pp. 14664-14673, 2020.

[39] M. U. Rothmann, W. Li, Y. Zhu et al., "Structural and chemical changes to $\mathrm{CH} 3 \mathrm{NH} 3 \mathrm{PbI}$ Induced by electron and gallium ion beams," Advanced Materials, vol. 30, no. 25, p. 1800629, 2018.

[40] Y. Li, W. Zhou, Y. Li et al., "Unravelling degradation mechanisms and atomic structure of organic-inorganic halide perovskites by cryo-EM," Joule, vol. 3, no. 11, pp. 2854-2866, 2019.

[41] Z. Dang, J. Shamsi, F. Palazon et al., "In situ transmission electron microscopy study of electron beam-induced transformations in colloidal cesium lead halide perovskite nanocrystals," ACS Nano, vol. 11, no. 2, pp. 2124-2132, 2017.

[42] N. Yi, S. Wang, Z. Duan, K. Wang, Q. Song, and S. Xiao, “Tailoring the performances of lead halide perovskite devices with electron-beam irradiation," Advanced Materials, vol. 29, no. 34, p. 1701636, 2017.

[43] J. Ran, O. Dyck, X. Wang, B. Yang, D. B. Geohegan, and K. Xiao, "Electron-beam-related studies of halide perovskites: challenges and opportunities," Advanced Energy Materials, vol. 10, no. 26, p. 1903191, 2020.

[44] Y. Yang, C. Gu, and J. Li, "Sub-5 nm metal nanogaps: physical properties, fabrication methods, and device applications," Small, vol. 15, no. 5, article e1804177, 2019.

[45] T. H. P. Chang, "Proximity effect in electron-beam lithography," Journal of Vacuum Science and Technology, vol. 12, no. 6, pp. 1271-1275, 1975.

[46] J. Liu, C. Gao, X. He et al., "Improved crystallization of perovskite films by optimized solvent annealing for high efficiency solar cell," ACS Applied Materials \& Interfaces, vol. 7, no. 43, pp. 24008-24015, 2015.

[47] E. Jokar, C.-H. Chien, A. Fathi, M. Rameez, Y.-H. Chang, and E. W.-G. Diau, "Slow surface passivation and crystal relaxation with additives to improve device performance and durability for tin-based perovskite solar cells," Energy \& Environmental Science, vol. 11, no. 9, pp. 2353-2362, 2018.

[48] J. Hu, C. Wang, S. Qiu et al., "Spontaneously self-assembly of a 2D/3D heterostructure enhances the efficiency and stability in printed perovskite solar cells," Advanced Energy Materials, vol. 10, no. 17, p. 2000173, 2020.

[49] N. Dong, X. Fu, G. Lian et al., "Solvent-assisted thermalpressure strategy for constructing high-quality $\mathrm{CH} 3 \mathrm{NH} 3 \mathrm{PbI} 3$ xClxFilms as high-performance perovskite photodetectors," ACS Applied Materials \& Interfaces, vol. 10, no. 10, pp. 83938398, 2018.

[50] C. Bi, Q. Wang, Y. Shao, Y. Yuan, Z. Xiao, and J. Huang, "Nonwetting surface-driven high-aspect-ratio crystalline grain growth for efficient hybrid perovskite solar cells," Nature Communications, vol. 6, no. 1, p. 7747, 2015.

[51] X. Zou, G. Zheng, Q. Yuan et al., "Imaging based on metalenses," PhotoniX, vol. 1, no. 1, p. 2, 2020.

[52] X. Zhu, C. Vannahme, E. Hojlund-Nielsen, N. A. Mortensen, and A. Kristensen, "Plasmonic colour laser printing," Nature Nanotechnology, vol. 11, no. 4, pp. 325-329, 2016.

[53] A. Kristensen, J. K. W. Yang, S. I. Bozhevolnyi et al., "Plasmonic colour generation," Nature Reviews Materials, vol. 2, no. 1, p. 16088, 2017.

[54] T. Zhan, J. Xiong, J. Zou, and S. T. Wu, "Multifocal displays: review and prospect," PhotoniX, vol. 1, no. 1, p. 10, 2020.

[55] L. Liu, J. A. McLeod, R. Wang, P. Shen, and S. Duhm, "Tracking the formation of methylammonium lead triiodide perovskite," Applied Physics Letters, vol. 107, no. 6, article 061904, 2015. 
[56] Q. Chen, C. Dwyer, G. Sheng et al., "Imaging beam-sensitive materials by electron microscopy," Advanced Materials, vol. 32, no. 16, article e1907619, 2020.

[57] J. Chen and N.-G. Park, "Materials and methods for interface engineering toward stable and efficient perovskite solar cells," ACS Energy Letters, vol. 5, no. 8, pp. 2742-2786, 2020.

[58] W. Xu, Y. Gao, W. Ming et al., "Suppressing defects-induced nonradiative recombination for efficient perovskite solar cells through green antisolvent engineering," Advanced Materials, vol. 32, no. 38, article 2003965, 2020.

[59] C. Zou, C. Chang, D. Sun, K. F. Bohringer, and L. Y. Lin, "Photolithographic patterning of perovskite thin films for multicolor display applications," Nano Letters, vol. 20, no. 5, pp. 3710-3717, 2020.

[60] S. J. Clark, M. D. Segall, C. J. Pickard et al., "First principles methods using CASTEP," Zeitschrift fuer Kristallographie, vol. 220, no. 5/6, p. 567, 2005.

[61] D. Vanderbilt, "Soft self-consistent pseudopotentials in a generalized eigenvalue formalism," Physical Review B, vol. 41, no. 11, pp. 7892-7895, 1990.

[62] M. C. Payne, M. P. Teter, D. C. Allan, T. A. Arias, and J. D. Joannopoulos, "Iterative minimization techniques for ab initio total-energy calculations: molecular-dynamics and conjugate gradients," Reviews of Modern Physics, vol. 64, no. 4, pp. 1045-1097, 1992.

[63] J. P. Perdew and Y. Wang, "Accurate and simple analytic representation of the electron-gas correlation-energy," Physical Review B, vol. 45, no. 23, pp. 13244-13249, 1992.

[64] H. J. Monkhorst and J. D. Pack, "Special points for Brillouinzone integrations," Physical Review B, vol. 13, no. 12, pp. 5188-5192, 1976. 